2007-01-01

\title{
Chlamydia Trachomatis Detection in Cervical PreservCyt Specimens From an Irish Urban Female Population
}

\author{
Helen Keegan \\ Technological University Dublin, helen.keegan@tudublin.ie \\ Fergus Ryan \\ Technological University Dublin, fergus.x.ryan@tudublin.ie \\ Alison Malkin \\ Technological University Dublin, alison.malkin@tudublin.ie
}

See next page for additional authors

Follow this and additional works at: https://arrow.tudublin.ie/scschbioart

Part of the Genetics Commons, and the Molecular Genetics Commons

\section{Recommended Citation}

Keegan, H. et al. (2007) Chlamydia trachomatis detection in cervical PreservCyt specimens from an Irish urban female population. Cytopathology. Apr2009, Vol. 20 Issue 2, p111-116. doi:10.1111/

j.1365-2303.2007.00534.x

This Article is brought to you for free and open access by the School of Biological Sciences at ARROW@TU Dublin. It has been accepted for inclusion in Articles by an authorized administrator of ARROW@TU Dublin. For more information, please contact arrow.admin@tudublin.ie, aisling.coyne@tudublin.ie,gerard.connolly@tudublin.ie. Funder: Strand III

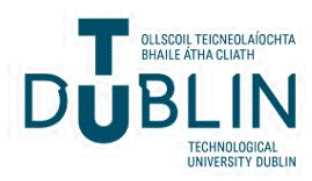




\section{Authors}

Helen Keegan, Fergus Ryan, Alison Malkin, M. Griffin, and Helen Lambkin

This article is available at ARROW@TU Dublin: https://arrow.tudublin.ie/scschbioart/43 


\title{
Chlamydia trachomatis detection in cervical PreservCyt specimens from an Irish urban female population
}

\author{
H. Keegan* ${ }^{\dagger}$, F. Ryan ${ }^{\dagger}$, A. Malkin ${ }^{\dagger}$, M. Griffin ${ }^{\dagger \S}$ and H. Lambkin ${ }^{\dagger}$ \\ *Department of Pathology, Coombe Women’s Hospital, `School of Biological Sciences, Dublin Institute of Technology, \\ tDepartment of Histopathology, St James’s Hospital and SDepartment of Histopathology, University of Dublin, Trinity College, \\ Dublin, Ireland
}

Accepted for publication 17 September 2007

H. Keegan, F. Ryan, A. Malkin, M. Griffin and H. Lambkin

Chlamydia trachomatis detection in cervical PreservCyt specimens from an Irish urban female population

Objective: The aim of this study was to determine the prevalence of cervical Chlamydia trachomatis infection by polymerase chain reaction (PCR) in urban women undergoing routine cervical cytological screening and to investigate the relationship with age, cytology, smoking status and concurrent human papillomavirus (HPV) infection.

Methods: A total of 996 women (age range 16-69 years) attending general practitioners for routine liquid-based cervical smear screening in the Dublin area were recruited in the study of prevalence of $C$. trachomatis. Informed consent was obtained and liquid-based cytology (LBC) specimens were sent for cytological screening. DNA was extracted from residual LBC and tested for C. trachomatis by PCR using the highly sensitive C. trachomatis plasmid (CTP) primers and for HPV infection using the MY09/11 primers directed to the HPV Ll gene in a multiplex format.

Results: The overall prevalence of $C$. trachomatis was $5.4 \%$. Prevalence was highest in the $<25$ years age group $(10 \%)$. Coinfection with HPV and C. trachomatis occurred in $1 \%$ of the screening population. A higher rate of smoking was observed in women positive for C. trachomatis, HPV infections or those with abnormal cervical cytology. Chlamydia trachomatis infection was not associated with abnormal cytology.

Conclusions: Women (5.4\%) presenting for routine cervical screening are infected with C. trachomatis. Opportunistic screening for C. trachomatis from PreservCyt sample taken at the time of cervical cytological screening may be a possible strategy to screen for C. trachomatis in the Irish female population.

Keywords: Chlamydia trachomatis, PreservCyt, cervical cytology, human papillomavirus, smoking, Irish

\section{Introduction}

Chlamydia trachomatis is the most common bacterial sexually transmitted infection (STI) worldwide with approximately 90 million cases occurring annually. ${ }^{1}$ Chlamydia trachomatis causes a variety of disease states ranging from asymptomatic infections, cervicitis and pelvic inflammatory disease to ectopic pregnancies and tubal infertility with each successive round of infection increasing the risk of serious

Correspondence:

Helen Keegan, Department of Pathology, Coombe Women's Hospital, Dublin 8, Ireland

Tel.: +353 1 4085674; Fax: +353 1 4085608;

E-mail: keeganh28@gmail.com sequelae. $^{2}$ High-risk human papillomavirus (HPV) infection of the cervix is necessary for the development of preneoplastic cervical lesions, which may be detected on Pap smear. ${ }^{3}$ Cigarette smoking and C. trachomatis infections are now considered independent risk factors for the development of cervical cancer. $^{4-6}$

In Ireland, the incidence of $C$. trachomatis infections is rising each year, with 2803 cases reported during 2004. ${ }^{7}$ Consequently, the need for C. trachomatis screening in Ireland is under review. ${ }^{8}$ Currently in the USA, Centre for Disease Control and Prevention recommends that women $<25$ years, women with multiple sexual partners, women having had a change in partner, women who have symptoms suggestive of chlamydial infection and those who have had a 
previous STI are screened at regular intervals. ${ }^{9}$ These recommendations have been translated into active screening programmes across all states, with welldocumented evidence of a reduction in prevalence in areas where intervention has been in place for a number of years. ${ }^{10}$ Similarly, in Sweden, a national C. trachomatis screening programme implemented in the 1980s has been associated with a dramatic reduction in incidence of $C$. trachomatis and its adverse sequelae. $^{11}$

Commercial nucleic acid-based C. trachomatis detection methods such as the Amplicor ${ }^{\circledR}$ CT/NG Test (RocheMolecular Systems, Branchburg, NJ, USA), the Digene hybrid capture (HCII; Digene, Gaithersburg, MD, USA) and the APTIMA Combo-2 assay (Genprobe Inc., San Diego, CA, USA) demonstrate both high sensitivities and specificities. ${ }^{12,13}$ They commonly target the C. trachomatis multicopy plasmid genes and are routinely performed on cervical swabs or urines. ${ }^{12,13}$ The use of molecular methods for the detection of high-risk HPV DNA and mRNA from PreservCyt cervical specimens has substantial potential and molecular testing for HPV has been proposed as an adjunct to cervical cytology in screening algorithms. ${ }^{14,15}$ Many studies have demonstrated the feasibility of screening PreservCyt specimens for detecting infections in the genital tract other than $\mathrm{HPV}^{16-18}$ and other studies have reported on the stability of nucleic acids in PreservCyt. ${ }^{19,20}$

The aim of this study was to determine the prevalence of $C$. trachomatis and HPV infections in Irish women attending their general practitioner (GP) for a cervical smear test. C. trachomatis infections were analysed based on age, smoking status, cervical cytology and coinfection with HPV.

\section{Methods}

\section{Study cohort}

The population consisted of 996 women who attended one of nine participating GPs in Dublin city and suburban areas for cervical smear testing over a period of 14 months between December 2003 and February 2005. Women were recruited to the study regardless of previous history or symptoms of disease. Women were invited to participate in the study of prevalence of C. trachomatis by the GP on receipt and understanding of an information leaflet and on completion of a consent form. Details of current cigarette smoking status, age and cytological diagnosis were obtained.
The study was anonymized and no patient identifiers were recorded.

\section{Ethical approval}

Ethical approval was obtained for the study from the St. James' Hospital Ethics Committee Review Board in August 2003.

\section{Specimen collection and processing}

Cervical specimens were taken and placed in a vial of PreservCyt (Cytyc Corporation, Marlborough, MA, USA) medium and transported to St. James' Cytology Laboratory where a cervical smear was prepared using the ThinPrep (Cytyc Corporation, USA) processor. Residual specimens were then kept at room temperature until DNA was extracted as described previously. ${ }^{21}$ Briefly, PreservCyt specimen (4 ml) was vortexed vigorously, then centrifuged at $3000 \mathrm{~g}$ and the pellet was washed twice with TE buffer $(10 \mathrm{mM}$ Tris, $1 \mathrm{mM}$ ethylenediaminetetraacetic acid, $\mathrm{pH}$ 8.0). Cell pellets were resuspended in TE buffer $(200 \mu \mathrm{l})$ and DNA was extracted using the QIAamp DNA Mini Kit (Qiagen Ltd, Crawley, UK) according to the manufacturer's instructions. Polymerase chain reaction (PCR) was performed using the CTP primers for detection of the $C$. trachomatis cryptic plasmid and MY09/11 primers for the detection of high and lowrisk HPV in a multiplex format as described previously. $^{22}$ The multiplex PCR included primers for amplification of human $\beta$-globin to ensure quality of the nucleic acid extraction.

\section{Statistical analysis}

Statistical data were analysed using SPSS version 11.0 software. Pearson Chi-square tests were performed to compare prevalence of $C$. trachomatis with age, smoking status, abnormal cytology and HPV coinfection.

\section{Results}

\section{Study population}

The age of the study population ranged from 16 to 72 years. The average age of women presenting for routine cervical screening was 35 years. Of the population studied, $187 / 996$ (19\%) were <25 years, $401 / 996(40 \%)$ were between the ages of 25 and 35 years and 408/996 (41\%) >35 years (Table 1$)$. 
Table 1. Prevalence of Chlamydia trachomatis in cervical PreservCyt specimens and age $(n=996)$

\begin{tabular}{llcc}
\hline & \multicolumn{3}{l}{ Age } \\
\cline { 2 - 4 } C. trachomatis & $<25$ & $25-35$ & $>35$ \\
\hline Positive & $18(10)$ & $20(5)$ & $16(4)$ \\
Negative & $169(90)$ & $381(95)$ & $392(96)$ \\
Total & 187 & 401 & 408 \\
\hline
\end{tabular}

Values are represented as $n(\%)$.

\section{Prevalence of C. trachomatis}

The overall prevalence of C. trachomatis was 5.4\%. Prevalence was $10 \%(18 / 187)$ in the age group of $<25$ years, $5 \%(20 / 401)$ in the $25-35$ years and $4 \%$ $(16 / 408)$ in the $>35$ years. Thirty-three per cent (18/54) of all C. trachomatis infections were in the $<25$ years age group, 37\% (20/54) in the 25-35 years age group and $30 \%(16 / 54)$ in the $>35$ years age group (Table 1). Cumulatively $70 \%(38 / 54)$ of $C$. trachomatis infections occurred in the $<35$ years age group. The trend of decreasing prevalence of $C$. trachomatis with age was highly significant $(P<0.0001)$.

\section{Coinfection with C. trachomatis and HPV}

Of the 54 C. trachomatis-infected specimens, 11 $(20.4 \%)$ also contained HPV. The overall coinfection rate within the population studied was $1 \%$. The average age of women infected with both organisms was 31 years with 8/11 concomitant infections occurring in women under 35 years of age.

\section{C. trachomatis and cervical cytology}

Of the 54 C. trachomatis-positive samples, 50 (92.6\%) had normal cytology, $2(3.7 \%)$ had borderline cytol- ogy and $2(3.7 \%)$ had cervical intraepithelial neoplasia grade I (CIN-I) lesions. C. trachomatis infection was not statistically associated with abnormal cytology.

\section{Smoking and cervical cytology}

Details of tobacco smoking were obtained for 706 of the 997 women in the study. Overall, 191/706 $(27.1 \%)$ of individuals admitted to smoking on a daily basis. However, no information on number of cigarettes or duration of smoking was available for this study. Of the 191 smokers, $19.4 \%$ had some degree of abnormal cytology, i.e. evidence of either borderline cytology or CIN lesions versus $7.4 \%$ of non-smokers (Table 2). Smoking was more common in women with abnormal cytology $(P<0.0001)$. The percentage of women within each category of abnormal cytology was higher for the smokers than the non-smokers (Table 2).

\section{Smoking and prevalence of $C$. trachomatis and HPV infections}

Of the women who smoked 46/191 (24\%) had HPV infections and 15/191 (8\%) had C. trachomatis infections. Of the non-smokers, $80 / 515$ (16\%) had HPV infections and 23/515 (4\%) had C. trachomatis infections (Table 3). Smoking was statistically associated with both HPV and $C$. trachomatis infections $(P=0.008$ and $P<0.001)$. Four of $191(2.1 \%)$ smokers were coinfected with HPV and C. trachomatis versus 2/515 $(0.4 \%)$ non-smokers (Table 3$)$.

\section{Discussion}

Screening for $C$. trachomatis may contribute to the prevention of pelvic inflammatory disease and reduce the cost of reproductive health problems,
Table 2. Smoking status and cytology result $(n=706)$

\begin{tabular}{|c|c|c|c|c|c|c|}
\hline \multirow[b]{2}{*}{ Smoking status } & \multicolumn{6}{|c|}{ Cervical cytology } \\
\hline & Normal & Abnormal $^{*}$ & Borderline $^{\dagger}$ & CIN-I & CIN-II & CIN-III \\
\hline Smoker $(n=191)$ & $154(80.6)$ & $37(19.4)$ & $16(8.4)$ & $15(7.8)$ & $3(1.6)$ & $3(1.6)$ \\
\hline Non-smoker $(n=515)$ & $477(92.6)$ & $38(7.4)$ & $15(2.9)$ & $16(3.1)$ & $6(1.2)$ & $1(0.2)$ \\
\hline Total $(n=706)$ & 631 & 75 & 31 & 31 & 9 & 4 \\
\hline
\end{tabular}

Values are represented as $n(\%)$.

*Classified as having either borderline cytology or cervical intraepithelial neoplasia (CIN).

${ }^{\dagger}$ Borderline nuclear changes, including atypical glandular and squamous cells of undetermined significance. 


\begin{tabular}{|c|c|c|c|c|c|c|}
\hline \multirow[b]{2}{*}{ Smoking status } & \multicolumn{2}{|l|}{ HPV } & \multicolumn{2}{|c|}{ C. trachomatis } & \multicolumn{2}{|l|}{ Coinfection } \\
\hline & Negative & Positive & Negative & Positive & Negative & Positive \\
\hline Smoker $(n=191)$ & $145(76)$ & $46(24)$ & $176(92)$ & $15(8)$ & $187(98)$ & $4(2)$ \\
\hline Non-smoker $(n=515)$ & $435(84)$ & $80(16)$ & $492(96)$ & $23(4)$ & $513(99.6)$ & $2(0.4)$ \\
\hline Total $(n=706)$ & 580 & 126 & 668 & 38 & 700 & 6 \\
\hline
\end{tabular}

Table 3. Smoking status, HPV and Chlamydia trachomatis infection and coinfection $(n=706)$

Values are represented as $n(\%)$.

HPV, human papillomavirus.

such as ectopic pregnancies and infertility. Before any effective screening programme is introduced into a population, it is necessary to determine the expected prevalence rate and identify those groups who should be targeted. Ireland is currently examining the need for a C. trachomatis screening programme. The aim of this study was to determine the prevalence of $C$. trachomatis in an urban female population undergoing routine opportunistic cervical screening.

In this study, a multiplex PCR was performed to screen samples simultaneously for HPV and C. trachomatis. The sensitivity and specificity of this assay was determined previously with respect to the commercially available HCII assay for HPV detection and the ligase chain reaction assay (LCx; Abbott Laboratories, Abbott Park, IL, USA) for C. trachomatis detection (results not shown). The sensitivity and specificity of the multiplex for the detection of HPV with respect to the HCII assay were $95 \%$ and $100 \%$, respectively. The sensitivity and specificity of the multiplex for the detection of C. trachomatis were $100 \%$ with respect to the commercial LCx assay. In our study, an overall prevalence of $C$. trachomatis of $5.4 \%$ was determined with a prevalence of $10 \%$ in the $<25$ years. It is widely known that the prevalence of $C$. trachomatis depends upon the setting, context and country studied. In 2002, the European Union BioMed Concerted Action Group undertook a systematic review of over 300 studies of prevalence of $C$. trachomatis among European women. The mode was $6 \%$ for women seeking contraception and $4 \%$ for women having cervical smears. ${ }^{23}$ Our finding of $5.4 \%$ in women having cervical smears correlates well with the European average. In a systematic literature review of costeffectiveness studies conducted between 1990 and 2000, screening for C. trachomatis was cost-effective at prevalences of $3.1-10 \%$ and cost saving at prevalences of $>1.1 \%$ if age was used as a selection factor and DNA-based tests on urine used. ${ }^{24}$ Based on these figures opportunistic screening in the Irish population would be cost-effective particularly for the $<25$ years.

STI surveillance in Ireland is mostly genitourinary medicine clinic based, with few incidence reports made from primary care settings. ${ }^{7} \mathrm{HPV}$ and C. trachomatis infections are among the most common cases of STI reported in Ireland. In 2004, the three most commonly reported STIs were ano-genital warts $(n=4174)$, C. trachomatis $(n=2803)$ and non-specific urethritis $(n=2746) .{ }^{7}$ Few studies have investigated the prevalence of $C$. trachomatis in the Republic of Ireland. In 2004, a study was conducted in the midwestern region to determine the prevalence of C. trachomatis in men. Of 562 men attending orthopaedic clinics and university sports facilities, 5.9\% were positive for C. trachomatis. ${ }^{25}$ Recently a study was conducted to determine the prevalence of $C$. trachomatis in women attending a maternity hospital for antenatal, fertility and family planning services. A prevalence of C. trachomatis of $3.7 \%$ was found in urine samples. ${ }^{26}$ Testing of urine samples using nucleic acid-based techniques has often been criticized due to the presence of amplification inhibitors in urine. ${ }^{27-29}$ In our study, DNA was extracted from residual cells in PreservCyt medium following routine cervical smear testing from which all samples amplified for the internal control and no amplification inhibition was observed. Our prevalence of $5.4 \%$ may be a truer estimate of $C$. trachomatis infections in the Irish female urban population regardless of parity or fertility status.

Previous studies have demonstrated a decrease in prevalence of $C$. trachomatis with age. ${ }^{30,31}$ This trend was observed in our study with incidence reducing from $10 \%$ in the $<25$ years age group to $5 \%$ in the $25-35$ years and $4 \%$ in the $>35$ years. The incidence of C. trachomatis infections was highest in the $<25$ years age group; however, this group is not commonly targeted for cervical screening. Since the majority of C. trachomatis infections are asymptomatic, 
testing of cervical PreservCyt samples may be a cost effective strategy for the screening of sexually active women.

In our study, those who smoked had a higher incidence of $C$. trachomatis and HPV infections than non-smokers. However, the strength of this association is limited by the lack of detailed information on number of cigarettes smoked per day and the duration of smoking. The higher incidence may be as a result of lifestyle factors linking high-risk sexual behaviour to unhealthy lifestyle choices. Other studies have also demonstrated a positive association of HPV and C. trachomatis infection with current smoking. ${ }^{32,33}$

Chlamydia trachomatis is now considered an independent risk factor for the development of cervical cancer. $^{34}$ A recent study on colposcopy patients reporting a prevalence of $3.4 \%$, suggested that routine screening for $C$. trachomatis be carried out in colposcopy clinics. ${ }^{35}$ In our study, $20.4 \%$ of C. trachomatis infected samples were coinfected by HPV; however, no association was seen between $C$. trachomatis infection and abnormal cytology. A recent study in Argentina found that prevalence of $C$. trachomatis was higher in HPV-infected cohorts. ${ }^{36}$ Chlamydia trachomatis-infected cohorts may also overlap with those infected by other STIs and identify those at increased risk of cervical neoplasia.

Numerous studies conducted in other countries have evaluated and advocated opportunistic C. trachomatis screening approaches in primary health care settings. ${ }^{37-39}$ While urine testing has been the mainstay in screening for C. trachomatis, liquid-based cytology affords the simultaneous evaluation of cytology, HPV and C. trachomatis from a single sample. Opportunistic screening for C. trachomatis at the time of cervical screening would not only identify women at risk for adverse reproductive complications but taken together with cytology result, HPV status, smoking status and other infecting STI identify those at higher risk for development of cervical neoplasia.

\section{Acknowledgments}

We wish to thank the GPs and women who participated in this study. We would also like to acknowledge the staff of the Cytology Laboratory in St. James' Hospital who aided in sample collection and Dr. John Kearney of the School of Biological Sciences, Dublin Institute of Technology for his assistance in statistical analyses. This work was funded by the Research
Support Unit, Dublin Institute of Technology under the Technological Sector- III grant of the National Development Plan.

\section{References}

1. Brunham RC, Rey-Ladino J. Immunology of Chlamydia infection: implications for a Chlamydia trachomatis vaccine. Nat Rev Immunol 2005;5:149-61.

2. Polyzos NP, Vittoraki A, Tsappi M et al. Food for thought in women's health. The gynecologist: the new European gatekeeper in Chlamydia trachomatis infection. Acta Obstet Gynecol Scand 2006;85:1156-9.

3. Munoz N, Castellsague X, de Gonzalez AB, Gissmann L. Chapter 1: HPV in the etiology of human cancer. Vaccine 2006;24:S1-10.

4. Berrington de Gonzalez A, Green J. Comparison of risk factors for invasive squamous cell carcinoma and adenocarcinoma of the cervix: collaborative reanalysis of individual data on 8,097 women with squamous cell carcinoma and 1,374 women with adenocarcinoma from 12 epidemiological studies. Int J Cancer 2007;120:885-91.

5. Madeleine MM, Anttila T, Schwartz SM et al. Risk of cervical cancer associated with Chlamydia trachomatis antibodies by histology, HPV type and HPV cofactors. Int J Cancer 2007;120:650-5.

6. Smith JS, Bosetti C, Munoz N et al. IARC multicentric case control study. Chlamydia trachomatis and invasive cervical cancer: a pooled analysis of the IARC multicentre case-control study. Int J Cancer 2004;111:431-9.

7. Health Protection Surveillance Centre. Sexually Transmitted Infections 2004 Annual Summary Report. Dublin, Ireland: HPSC; 2005: pp. 1-18.

8. Health Protection Surveillance Centre. The Need for Chlamydia Screening in Ireland. Dublin, Ireland: HPSC; 2005.

9. Centers for Disease Control and Prevention, USA. Recommendations for the prevention and management of Chlamydia trachomatis infections, 1993. MMWR Recomm Rep 1993;42:1-39.

10. Centers for Disease Control and Prevention, USA. Sexually Transmitted Diseases Surveillance 2000 Supplement, Chlamydia prevalence monitoring project. Atlanta, GA: US Department of Health and Human Services, Centers for Disease Control and Prevention; November 2001.

11. Kamwendo F, Forslin L, Bodin L, Danielsson D. Programmes to reduce pelvic inflammatory disease - the Swedish experience. Lancet 1998;351:25-8.

12. Darwin LH, Cullen AP, Crowe SR et al. Evaluation of the hybrid capture $2 \mathrm{CT} / \mathrm{GC}$ DNA tests and the GenProbe PACE 2 tests from the same male urethral swab specimen. Sex Transm Infect 2002;29:576-80.

13. Lowe P, O'Loughlin P, Evans K et al. Comparison of the Gen-Probe APTIMA Combo 2 assay to the AMPLICOR CT/NG assay for detection of Chlamydia trachomatis and 
Neisseria gonorrhoeae in urine samples from Australian men and women. J Clin Microbiol 2006;44:2619-21.

14. Cox T, Cuzick J. HPV DNA testing in cervical cancer screening: from evidence to policies. Gynecol Oncol 2006;103:8-11.

15. Lie AK, Risberg B, Borge B et al. DNA- versus RNA-based methods for human papillomavirus detection in cervical neoplasia. Gynecol Oncol 2005;97:908-15.

16. Zhang W, Cohenford M, Lentrichia B et al. Detection of Chlamydia trachomatis by isothermal ramification amplification method: a feasibility study. J Clin Microbiol 2002;40:128-32.

17. Koumans EH, Black CM, Markowitz LE et al. Comparison of methods for detection of Chlamydia trachomatis and Neisseria gonorrhoeae using commercially available nucleic acid amplification tests and a liquid pap smear medium. $J$ Clin Microbiol 2003;41:1507-1 1.

18. Hopwood J, Mallinson H, Hodgson E, Hull L. Liquid based cytology: examination of its potential in a chlamydia screening programme. Sex Transm Infect 2004;80:371-3.

19. Sailors J, Gander R, Saboorian MH et al. Stability of PreservCyt for hybrid capture (HC II) HPV test. Diagn Cytopathol 2005;32:260-3.

20. Castle PE, Solomon D, Hildesheim A et al. Stability of archived liquid-based cervical cytologic specimens. Cancer 2003; 99:89-96.

21. Keegan H, Boland C, Malkin A et al. Comparison of DNA extraction from cervical cells collected in PreservCyt solution for the amplification of Chlamydia trachomatis. Cytopathology 2005;16:82-7.

22. Keegan H, Malkin A, Griffin M et al. Validation of a multiplex PCR assay for the simultaneous detection of human papillomavirus and Chlamydia trachomatis in cervical PreservCyt samples. Clin Chem 2005;51:1301-2.

23. Wilson JS, Honey E, Templeton A et al. EU Biomed Concerted Action Group. A systematic review of the prevalence of Chlamydia trachomatis among European women. Hum Reprod Update 2002;8:385-94.

24. Honey E, Augood C, Templeton A et al. Cost effectiveness of screening for Chlamydia trachomatis: a review of published studies. Sex Transm Infect 2002;78:406-12.

25. Powell J, O'Connor C, O'harlaithe M, De Freitas J. Chlamydia trachomatis prevalence in men in the mid-west of Ireland. Sex Transm Infect 2004;80:349-53.

26. McMillan HM, O'Carroll H, Lambert JS et al. Screening for Chlamydia trachomatis in asymptomatic women attending outpatient clinics in a large maternity hospital in Dublin, Ireland. Sex Transm Infect 2006;82:503-5.

27. Jones S, Barker S, Athan E, Graves S. The tip of the iceberg: opportunistic screening for Chlamydia trachomatis in asymptomatic patients attending a young people's health clinic reveals a high prevalence - a pilot study. Sex Health 2004;1:115-9.

28. Mahony J, Chong S, Jang D et al. Urine specimens from pregnant and nonpregnant women inhibitory to amplification of Chlamydia trachomatis nucleic acid by PCR, ligase chain reaction, and transcription-mediated amplification: identification of urinary substances associated with inhibition and removal of inhibitory activity. $J$ Clin Microbiol 1998;36:3122-6.

29. Shrier LA, Dean D, Klein E et al. Limitations of screening tests for the detection of Chlamydia trachomatis in asymptomatic adolescent and young adult women. Am J Obstet Gynecol 2004;190:654-62.

30. van den Hoek JA, Mulder-Folkerts DK, Coutinho RA et al. Opportunistic screening for genital infections with Chlamydia trachomatis among the sexually active population of Amsterdam. II Over 90\% participation and almost 5\% prevalence. Ned Tijdschr Geneeskd 1999;143: 668-72.

31. Adams EJ, Charlett A, Edmunds WJ, Hughes G. Chlamydia trachomatis in the United Kingdom: a systematic review and analysis of prevalence studies. Sex Transm Infect 2004;80:354-62.

32. Boardman LA, Stanko C, Weitzen S, Sung CJ. Atypical squamous cells of undetermined significance: human papillomavirus testing in adolescents. Obstet Gynecol Surv 2005;60:435-7.

33. Zbroch T, Knapp P, Blonska E et al. Life style, Chlamydia trachomatis infection, bacterial vaginosis and their impact on the frequency of cervical lesions. Ginekol Pol 2004; 75:538-44.

34. Franceschi S. The IARC commitment to cancer prevention: the example of papillomavirus and cervical cancer. Recent Results Cancer Res 2005;166:277-97.

35. Tayal SC, Robertson A, Doxford L. An audit of Chlamydia trachomatis screening in colposcopy - Hartlepool experience. Int J STD AIDS 2005;16:500-1.

36. Golijow CD, Abba MC, Mouron SA et al. Chlamydia trachomatis and human papillomavirus infections in cervical disease in Argentine women. Gynecol Oncol 2005;96:181-6.

37. Harris DI. Implementation of chlamydia screening in a general practice setting: a 6-month pilot study. J Fam Plann Reprod Health Care 2005;31:109-12.

38. Yu MC, Li LH, Tang LH, Chen KT. Genital chlamydial infection among male attendees at a sexually transmitted disease clinic in urban Taiwan. Public Health 2007; 121:534-9.

39. Marrazzo JM, Ellen JM, Kent C et al. Acceptability of urine-based screening for Chlamydia trachomatis to asymptomatic young men and their providers. Sex Transm Dis 2007;34:147-53. 